\title{
FUNCTIONAL IMPROVEMENT OF THE PATIENTS AFTER HIP AND KNEE ARTHROPLASTY SURGERY, SHOULDER AND LUMBAR SPINE SURGERY
}

Golež Ana, MD, PhD, Department of Medical Rehabilitation, General and Teaching Hospital Celje, Slovenia

Introduction: Due to the aging population, there is an increasing number of patients who undergo total hip or knee replacement, shoulder or lumbar spine surgery, mostly because of a degenerative disease. If a patient's functional status after surgery is not optimal, he is referred to an appropriate rehabilitation programme.

Purpose: To evaluate the functional improvement of the patients after surgery, who participated the two-week rehabilitation programme.

Method: Functional status of the patients was evaluated, using Oswestry Disability Index for patients after lumbar spine surgery, Harris Hip Score for patients after hip arthroplasty surgery, Knee Society Score for patients after knee arthroplasty surgery and UCLA Shoulder Score for patients after shoulder surgery, at the beginning and at the end of the rehabilitation programme, and improvement was calculated.

Results: From September to December 201570 patients after knee and 95 after hip arthroplasty surgery, 42 after shoulder and 81 after lumbar spine surgery participated in a two-week rehabilitation programme. At the end of the rehabilitation Oswestry Disability Index improved for 8.1, Harris Hip Scores for 18.3, Knee Society Score for 36 and UCLA Shoulder Score for 6.7 points. The majority of the patients were independent in activities of daily living at the end of the rehabilitation programme.

Conclusions: Results show a good improvement of functional outcome of patients after knee and hip arthroplasty surgery, shoulder and lumbar spine surgery after a two-week rehabilitation programme; patients were mostly independent in the tasks of daily living. It is advisable that all patients with difficulties performing activities of daily living after surgery are referred to an appropriate rehabilitation programme. 